\title{
¿Los conocimientos criminológicos modulan la actitud punitiva?
}

\author{
Elisa GARCÍA EsPAÑA \\ Universidad de Málaga \\ Elisa@uma.es
}

Recibido: $19 / 11 / 2012$

Aceptado: 23/01/2013

\begin{abstract}
Resumen
El objetivo es demostrar cómo las actitudes punitivas más rigurosas suelen tener su origen en el desconocimiento de las funciones y del funcionamiento del sistema penal. Se parte de la hipótesis de que el conocimiento de la realidad delictiva, del delincuente y de la reacción penal hacen que las exigencias punitivistas sean menores que cuando se tenía un conocimiento estereotipado de dicha realidad. Para ello se ha pasado una encuesta al inicio y finalización del semestre a un grupo de tratamiento, formado por los alumnos de $1^{\circ}$ del Grado de Criminología, y un grupo de control compuesto por alumnos de $1^{\circ}$ de Derecho de la misma Universidad. Los resultados muestran que los conocimientos adquiridos producen un cambio significativo en su actitud punitiva, reduciéndose ésta con respecto a su actitud al inicio del curso. Palabras clave: Actitud punitiva, conocimientos, delincuencia, sistema de justicia penal
\end{abstract}

\section{Can Criminological Knowledge Modulate Punitive Attitude?}

\begin{abstract}
The aim is to show how punitive attitudes usually arise from ignorance of the functions and operation of the criminal justice system. We start from the assumption that knowledge of reality criminal, of offender and of criminal reaction make that punitive demands are lower than when you had a stereotyped knowledge of that reality. This has been a survey at the beginning and end of the semester to a treatment group, formed by the students of 1st Grade of Criminology, and a control group composed of students of 1st Law of the same University. The results show that students change their punitive attitude when their have deep understanding of the reality of crime, reducing their punitive attitude with respect to their attitude al the beginning of the semester.
\end{abstract}

Keywords: Punitive attitude, knowledge, delinquency, criminal justice system

\section{Referencia normalizada}

GARCÍA ESPAÑA, ELISA (2013): “Los conocimientos criminológicos modulan la actitud punitiva?”. Estudios sobre el mensaje periodístico. Vol. 19, Núm. especial abril, págs.: 753-761. Madrid, Servicio de Publicaciones de la Universidad Complutense.

Sumario: 1. Introducción; 1.1. Fundamentación teórica; 1.2. Contexto histórico. 2. Metodología, 2.1. Objetivos e hipótesis; 2.2. Diseño de la investigación; 2.3. Muestra; 2.4. instrumento de recogida de datos; 2.5. Validez de la muestra. 3. Desarrollo; 3.1. Conocimientos criminológicos; 3.2. Actitudes punitivas; 3.3. De las generalidades a lo concreto. 4. Conclusiones. 5. Referencias bibliográficas.

\section{Introducción}

\subsection{Fundamentación teórica}

Existen estereotipos sociales sobre la magnitud y evolución de la delincuencia en España, así como sobre el funcionamiento de la administración de justicia, que se retroalimentan a través de los medios de comunicación y de la clase política. Tanto unos como la otra aportan información (Martínez Rodríguez, 2012) y opiniones que dan la sensación de que la delincuencia en España es cada vez más numerosa, que los jóvenes son cada vez más violentos, los inmigrantes son más delincuentes, que las penas 
en España son blandas, que el sistema judicial es injusto, que los presos salen antes de tiempo de la cárcel, etc.

Estas percepciones a pie de calle (Mut Camacho, 2012) chocan frontalmente con un análisis científico y sereno de la realidad: Encuestas de victimización apuntan a un descenso de la delincuencia (García España y Díez Ripollés, 2009), las investigaciones sobre delincuencia juvenil no muestran un aumento alarmante de comportamientos delictivos entre jóvenes (Fernández Molina y otros, 2009), estudios sobre inmigrantes desmienten que ellos están más implicados en el delito que los nacionales (García España, 2001; Gómez Fraguela, 2009), las evaluaciones de programas de tratamientos en delincuentes sexuales concluyen en unas tasas de reincidencia más bajas que en delincuentes contra la propiedad (Redondo Illesca, 2006), las penas en España son las más altas de Europa, las cárceles están masificadas porque los presos entran pero no salen (García España y Díez Ripollés, 2012), etc.

Por otra parte, cuando se piensa en un "delincuente" se suele acudir al estereotipo de delincuente que nos presentan los medios de comunicación, que suelen ser violentos y peligrosos. Pero este tipo de sucesos, tan frecuentemente recogidos por los medios de comunicación, representan un porcentaje muy bajo de nuestra delincuencia. La mayoría de la delincuencia suele ser más leve y contra el patrimonio o la salud pública (vulgarmente conocido como delitos de droga).

El objetivo del presente artículo es mostrar que los conocimientos criminológicos permiten un cambio de actitud con respecto al punitivismo. Se parte de la hipótesis de que el conocimiento de la realidad delictiva, del delincuente y de la reacción penal hacen que las exigencias punitivistas sean menos rigoristas que cuando se tenía un conocimiento estereotipado de dicha realidad. Además, se ha querido recoger ambos tipos de delincuencia (violenta y contra el patrimonio) para comprobar también si la actitud punitiva queda matizada por la gravedad de los hechos.

Para todo ello se ha pasado una encuesta al inicio y finalización del semestre a un grupo de tratamiento, formado por los alumnos de $1^{\circ}$ del Grado de Criminología de la Universidad de Málaga, y un grupo de control compuesto por alumnos de $1^{\circ}$ de Derecho de la misma Universidad. Los resultados muestran que, siendo ambos grupos homogéneos, los conocimientos adquiridos por los alumnos de Criminología atendiendo a un adecuado proceso de comunicación por parte de la docente (Guanipa-Pérez, 2012) produce un cambio significativo en su actitud punitiva, reduciéndose ésta con respecto a su actitud al inicio del curso. Con todo, es posible confirmar que las encuestas meditadas, estos es, las que se realizan tras una profundización en la realidad sobre la que se ha de prestar opinión, difieren considerablemente de las opiniones espontáneas mediatizadas por conocimientos estereotipados (Marteache, 2010).

\subsection{Contexto histórico}

El Grado en Criminología es uno de los nuevos títulos que han sido creados al albur del Espacio Europeo de Enseñanza Superior en 2010. Sin embargo la Criminología como disciplina tiene un mayor bagaje en la historia reciente de España. De hecho la Criminología española desde los años 80 hasta la actualidad ha ido lentamente consolidándose, gracias al empeño de algunos académicos por hacer investigaciones em- 
píricas en este ámbito y a pesar de su dispersión entre diferentes disciplinas como la Psicología, Sociología, Derecho penal, Antropología, etc (Barberet, 2005). Pero los antecedentes de la Criminología en España son aún más remotos, pudiéndonos remontar al siglo XIX con Mariano Cubí i Soler (1801-1875) quien propuso un conjunto de ideas que anticipaban a las que más tarde desarrollara Lombroso. Los estudios realizados por autores como Rafael Salillas (1854-1923), Pedro Dorado Montero (18611919), Concepción Arenal (1820-1894) y Constancio Bernardo de Quirós (1873-1959) iniciaron una tradición de investigación en Criminología en España conocida como correccionalismo, esto es, la idea de la educación supervisada a los presos. Tal fue el calado de estos autores a nivel internacional que la obra de Dorado Montero fue una de las que inspiraron a los fundadores de la renombrada Escuela de Chicago en 1920.

Durante el siglo XIX y las dos primeras décadas del siglo XX instituciones como el Ateneo y la Institución Libre de Enseñanza fueron centros educativos pioneros. Pero con la guerra civil española (1936-39) prácticamente todos los criminólogos prestigiosos españoles se vieron obligados al exilio, desde donde continuaron sus trabajos. Durante los primeros años de la dictadura franquista, la criminología en España era prácticamente limitada a los esfuerzos aislados de algunos investigadores. Sin embargo, la década de 1950 trajo consigo un resurgimiento, influenciado por las nuevas ideas provenientes de Europa. La academia criminológica comenzó en las grandes ciudades como respuesta a la creciente preocupación pública por los problemas de seguridad urbana. En 1955 Octavio Victoria Pérez fundó el Instituto de Criminología en Barcelona inspirado en el modelo de estudios de justicia penal en los EE.UU. Y en 1964 se fundó el Instituto de Criminología de la Universidad Complutense se Madrid (Cerezo Domínguez, 2012).

A pesar de los antecedentes referidos se constata que la Criminología y la función de los criminólogos son grandes desconocidos en nuestra sociedad según se aprecia a través de los medios de comunicación, de la opinión pública y de la ausencia de salidas profesionales.

En los medios de comunicación la Criminología se identifica con la Criminalística, y la labor del criminólogo suele limitarse a tareas de policía científica. Series de televisión como CSI pueden haber contribuido a esta imagen distorsionada que la prensa y los programas de televisión y radio reproducen. Si este desconocimiento sobre la Criminología se une al hecho de que las decisiones políticas se toman a partir de la presión de las víctimas (Cerezo Domínguez, 2010) sin contar con la voz experta (Díez Ripollés, 2003), es comprensible el aumento constante de penas en el Código penal español como respuesta al problema de la delincuencia. Sin embargo, los criminólogos sabemos que se trata de posturas populistas y distanciadas de la eficacia y la eficiencia que en el marco de una política criminal bienestarista requiere la pacificación social.

\section{Metodología}

\subsection{Objetivos e hipótesis}

El objetivo de este trabajo es conocer si los sujetos pueden cambiar su opinión sobre los tipos de castigo a imponer una vez que conocen la eficiencia de los diversos tipos de sanciones, la verdadera capacidad de reinserción de los infractores y el funciona- 
miento y funciones del sistema penal. La hipótesis de partida es que a mayor conocimiento sobre la eficiencia del sistema penal menor actitud punitiva y viceversa.

\subsection{Diseño de la investigación}

Para verificar esta hipótesis se llevó a cabo el pase de una encuesta el primer día de clase presencial a alumnos de $1^{\circ}$ curso de los Grados de Derecho y Criminología (septiembre 2011) de la Universidad de Málaga.

Los alumnos del Grado de Criminología (grupo de tratamiento) tuvieron un temario directamente relacionado con la eficiencia del sistema penal, y se les mandaba información a través de la plataforma virtual y de una página de Facebook diseñada ad hoc para ellos.

Los alumnos del Grado de Derecho (grupo de control) no recibieron ningún tipo de instrucción en el ámbito penal y criminológico, siendo la asignatura en la que se pasaron las encuestas la de Filosofía del Derecho.

Al finalizar el $1^{\circ}$ semestre se hizo el segundo pase de la encuesta (enero 2012). Los asistentes el primer día de curso podían no ser los mismos que los que se encontraran en el aula al finalizar el semestre, debido a que algunos se incorporaron tarde, otros dejaron la carrera al poco de empezar, etc. Para controlar que los alumnos del $2^{\circ}$ pase fueran los mismos que los del $1^{\circ}$, se incluyó una pregunta al principio del cuestionario donde debían contestar si habían realizado o no a principio de curso esta misma encuesta. Los cuestionarios cuya primera pregunta había sido respondida negativamente se rechazaron del estudio. De esta manera podíamos observar los cambios de opinión en los dos grupos tras la formación recibida durante el primer semestre por el grupo de tratamiento (alumnos de $1^{\circ}$ Grado de Criminología).

\subsection{Muestra}

Nuestra muestra de conveniencia fue de 174 alumnos: 57 del Grado de Criminología, y 117 del Grado de Derecho. Para pasar las encuestas a los alumnos de $1^{\circ}$ del Grado de Derecho hubo que contar con la colaboración del responsable del grupo de la mañana de la asignatura de Filosofía de Derecho.

\subsection{Instrumento de recogida de datos}

El cuestionario que los alumnos respondieron de forma voluntaria y anónima consta de 34 preguntas genéricas sobre delincuencia, delincuentes, sistema judicial, prisiones y aspectos legales de ámbito penal. De todas ellas, 19 eran preguntas de conocimiento y las 15 restante de opinión.

Las preguntas de conocimiento estaban compuestas por 9 preguntas más técnicas, relacionadas con la punibilidad de menores de 14 años o con el principio de in dubio pro reo, y las 10 restantes obedecen a mitos existentes sobre la delincuencia en España y la eficiencia del sistema penal. Todas las preguntas de conocimiento se puntuaron con 1 ó 0 , siendo 1 el valor asignado a las respuestas acertadas, y representando el 0 lo contrario. De esta manera es posible observar si los cambios en el conocimiento producen cambios en la actitud punitiva de los sujetos, valorada ésta a partir de las restantes 15 preguntas. En ellas había preguntas relacionadas con delitos graves y 
delincuentes peligrosos, así como un supuesto práctico de un delito (entrar en un domicilio y robar un televisor, siendo el autor reincidente). Inspirada en el modelo CATSO, las 4 posibles respuestas ("totalmente de acuerdo", "bastante de acuerdo", "bastante en desacuerdo" y "totalmente en desacuerdo") a estas 15 preguntas se evaluaron de 1 a 4. La escala nos da un rango de 15 a 60. A mayor puntuación, más de acuerdo se está con la afirmación.

\subsection{Validez de la muestra}

Para que los resultados tengan validez y coexistencia los dos grupos (tratamiento y control) debían ser homogéneos. El perfil de los alumnos de Criminología y Derecho elegidos mostraron dicha homogeneidad ya que hay una proporción parecida de mujeres y hombres, en ambos Grados más del 80\% se sitúa entre los 17 y 19 años, la mayoría vive en poblaciones superiores a 50 mil habitantes, en ambos grupos la opción política mayoritaria es "centro", siendo los que se consideran de izquierdas un $33,3 \%$ en Criminología y un 25,4\% en Derecho, el $90 \%$ en ambos Grados proceden de Bachillerato como formación previa y desafortunadamente una gran mayoría no lee prensa no deportiva a diario, sino solo "a veces" (66,7\% en Criminología y $78 \%$ en Derecho).

\section{Desarrollo}

\subsection{Conocimientos criminológicos}

En el primer pase de la encuesta ambos grupos presentan escasos conocimientos de la realidad criminológica y de la política criminal española. De hecho, y tomando como referencia aquellas preguntas que son estadísticamente significativas, se observa que en ambos grupos el porcentaje de alumnos que responde afirmativamente a afirmaciones estereotipadas es mayoritario en cuestiones tales como "la delincuencia está en continuo aumento en España", "buena parte de la delincuencia es violenta", "el Código penal español es el menos represivo de Europa", "las penas de prisión en España son las más largas de Europa", "las penas de prisión se cumplen solo en parte", "los delincuentes sexuales son los más reincidentes", "el único fin de la pena es que el delincuente pague lo que ha hecho" y que "más de la mitad de los presos reinciden".

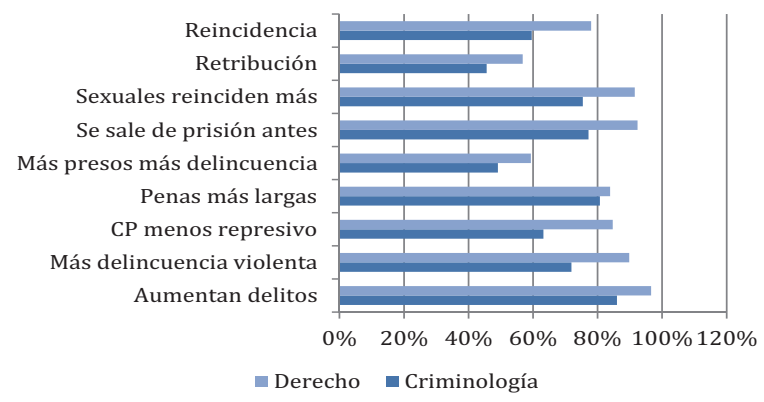

Gráfico 1: Porcentaje de desconocimiento $1^{\circ}$ pase (elaboración propia)

En el $2^{\circ}$ pase las respuestas de ambos grupos son muy distintas. Mientras que los estudiantes de Criminología aumentan considerablemente sus conocimientos en todas 
cuestiones evaluadas, salvo en la cuestión de si más de la mitad de los presos reinciden, los estudiantes de Derecho siguen manteniendo escasos conocimientos sobre la realidad delictiva española, respondiendo como correctas afirmaciones que recogen estereotipos frecuentes. No obstante, estos alumnos parecen haber adquirido conocimientos en principio que rigen nuestro sistema penal como son los referentes a la presunción de inocencia y al principio de reinserción social como fin principal al que se orienta la pena de prisión. En el siguiente gráfico se exponen los resultados del $2^{\circ}$ pase en los grupos de control y tratamiento. El porcentaje de conocimiento se representa con signos positivos, mientras que el porcentaje de alumnos que desconocen esa cuestión se representa en negativo en el lado opuesto en el gráfico.

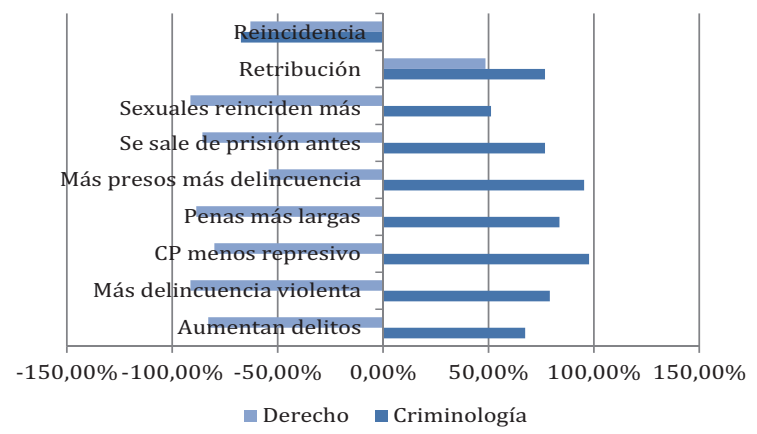

Gráfico $n^{\circ} 2$ : Porcentaje de conocimiento $2^{\circ}$ pase (Elaboración propia)

\subsection{Actitudes punitivas}

En cuanto a la actitud punitiva, en el primer pase ambos grupos presentan opiniones punitivas rigoristas -como se observa en el gráfico $\mathrm{n}^{\circ} 3$ - que se deducen de las opiniones mayoritarias de los estudiantes ante afirmaciones tales como "las penas en España son muy blandas", "la Ley penal del menor es blanda", "hay que restringir los permisos de salida y los terceros grados de los presos", "necesidad de cadena perpetua para los delincuentes peligrosos" y de "más disciplina en prisión". La hipótesis de partida es que estas opiniones punitivas traen causa de los conocimientos estereotipados que mostramos infra tanto en el grupo de tratamiento (estudiantes de Criminología) como en el grupo de control (estudiantes de Derecho).

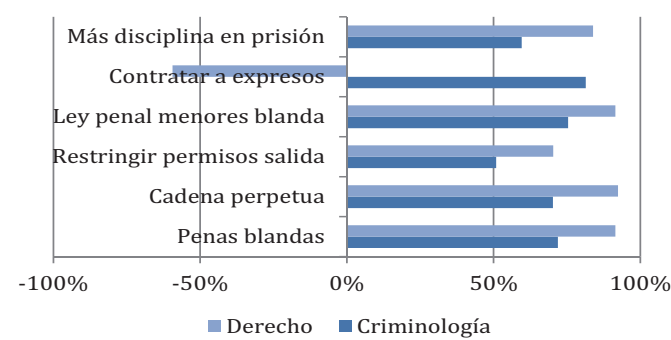

Gráfico $n^{\circ} 3$ : Actitudes punitivas $1^{\circ}$ pase (Elaboración propia) 


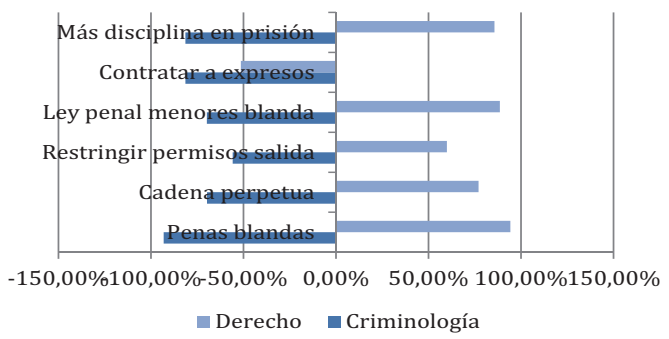

Gráfico $\mathrm{n}^{\circ} 4$ : Actitudes punitivas $2^{\circ}$ pase (Elaboración propia)

Sin embargo, en el segundo pase se observan cambios sustanciales de opinión en el grupo de estudiantes de Criminología (grupo de tratamiento que fue sometido a una formación especializada en esta materia), manteniéndose una actitud punitiva entre los estudiantes de Derecho que no se formaron específicamente en esta materia. En el gráfico $\mathrm{n}^{\mathrm{o}} 4$ se observa que un alto porcentaje de alumnos de Criminología no comparten las afirmaciones expuestas en el $2^{\circ}$ pase de la encuesta, mientras que la mayoría de los estudiantes de Derecho mantienen la misma actitud que en el primer pase, si bien, el porcentaje de alumnos es inferior en el segundo pase que en el primero (compruébese comparando los gráficos $\mathrm{n}^{\mathrm{o}} 3 \mathrm{y} \mathrm{n}^{\circ} 4$ ).

\subsection{De las generalidades a lo concreto}

En términos generales y con respecto a delincuentes peligrosos, los alumnos con conocimientos estereotipados presentan una actitud punitiva rigorista, según los expuesto infra. No obstante se quiso profundizar en la actitud punitiva de los estudiantes poniéndoles un supuesto práctico. Al final de cada uno de los dos pases de encuestas se les pedía a los alumnos que opinaran sobre qué pena merecía un joven de 20 años que se le encuentra culpable de robar en una vivienda por segunda vez, llevándose en esta ocasión un televisor. Además se les preguntó sobre qué pena consideraban que impondría un juez español. Las opciones de respuestas eran 8: multa, prisión de menos de 6 meses, prisión de 6 meses a 2 años, prisión de 2 a 5 años, prisión de más de 5 años, trabajo en beneficio de la comunidad (TBC), libertad condicional y otra (opción abierta). En la tabla $n^{\circ} 1$ se exponen las respuestas que obtuvieron el porcentaje de frecuencia más alto en los dos grupos objetos de estudio en ambos pases tanto en la pregunta de opinión (qué pena le pondría el encuestado) como en la de conocimiento (la pena que pondría el juez).

Tabla $\mathrm{n}^{\circ}$ 1: Cambios de conocimiento y opinión respecto a un robo de TV en vivienda por reincidente

\begin{tabular}{|l|c|c|c|c|}
\cline { 2 - 5 } \multicolumn{1}{c|}{} & \multicolumn{2}{c|}{ Criminología } & \multicolumn{2}{c|}{ Derecho } \\
\cline { 2 - 5 } \multicolumn{1}{c|}{} & Opinión & Conocim. & Opinión & Conocim. \\
\hline $1^{\circ}$ pase & $\mathbf{3 5 , 1 \%}$ & $\mathbf{3 1 , 6 \%}$ & $\mathbf{3 6 , 4 \%}$ & $\mathbf{3 5 , 9 \%}$ \\
& $6 \mathrm{~m}$ a 2 años & -6 meses & $6 \mathrm{~m}$ a 2 años & -6 meses \\
\hline $2^{\circ}$ pase & $\mathbf{3 7 , 2 \%}$ & $\mathbf{4 1 , 9 \%}$ & $\mathbf{4 0 \%}$ & $\mathbf{3 1 , 4 \%}$ \\
& TBC & $6 \mathrm{~m}$ a 2 años & $6 \mathrm{~m}$ a 2 años & -6 meses \\
\hline
\end{tabular}

Los datos de la tabla nos arrojan un resultado claro. La mayoría de los alumnos creían (gráfico $1,1^{\circ}$ pase) que los jueces imponía una pena menor de la que en realidad 
ponen para un caso de este tipo. Sin embargo, al terminar el semestre el 41,9\% de los alumnos de Criminología demuestran conocimientos correctos con respecto a la pena judicial a imponer, en contraposición con los alumnos de Derecho que aún siguen pensando que la pena que los jueces ponen es inferior. Además, se observa que la mayoría de los alumnos de Criminología varían de opinión del $1^{\circ}$ al $2^{\circ}$ pase con relación a la pena que ellos consideran que se debería imponer, siendo más elevada en el $1^{\circ}$ pase (pena de prisión de 6 meses a 2 años) que en el $2^{\circ}$ pase, que se inclinan por "trabajos en beneficio de la comunidad". Esto, por el contrario, no ocurre con los alumnos de Derecho, que en su mayoría mantienen la misma opinión en los dos pases de encuesta.

\section{Conclusiones}

Los datos que nos aportan las encuestas espontánea $\left(1^{\circ}\right.$ pase $)$ y meditada $\left(2^{\circ}\right.$ pase solo para el grupo de tratamiento) confirman las hipótesis de partida. Es cierto que la opinión pública, representada por los estudiantes de Criminología y Derecho, tiene una actitud punitiva rigorista que ante sucesos graves y atroces se concreta en demanda de reformas de las leyes penales para que éstas sean más duras con los delincuentes. Por otra parte, se observa que en la encuesta espontánea $\left(1^{\circ}\right.$ pase $)$ los conocimientos sobre la verdadera extensión de la delincuencia y la reacción penal existente son muy escasos. Por el contrario, en la encuesta meditada ( $2^{\circ}$ pase) la actitud punitiva del grupo de tratamiento se vuelve menos rigorista que al inicio del semestre y en comparación con las opiniones del grupo de control. Este mantenía una actitud punitiva similar a la del inicio del semestre. Igualmente los conocimientos del grupo de tratamiento aumentaron considerablemente con respecto al inicio del semestre y también con respecto al grupo de control, al cual no se le formó especialmente en la extensión de la delincuencia y la reacción penal frente a ella.

Sería interesante profundizar en la correlación existente con variables de interés como el sexo o la opción política. No obstante, dado que en esta investigación ambos grupos eran homogéneos entre sí y en ambos pases de la encuesta, es posible afirmar que los cambios de actitud del grupo de tratamiento trae su origen en el aumento de conocimientos adquiridos. Es más, que el cambio se produce no solo con respecto a la delincuencia más grave, que tal vez es la que se presenta más estereotipada ante la opinión pública, sino incluso con respecto a delitos contra el patrimonio no tan graves.

\section{Referencias bibliográficas}

BARBERET, Rosemary (2005): Spain, en European Journal of Criminology, Vol. 2 (3). Disponible en : http://euc.sagepub.com/cgi/content/abstract/2/3/341 [Consultado por última vez: 17 de septiembre de 2012]

CEREZO DOMÍNGUEZ, Ana Isabel (2010): El protagonismo de las víctimas en la elaboración de las leyes penales. Valencia, Tirant lo Blanch.

CEREZO DOMÍNGUEZ, Ana Isabel (2012): Criminology in Spain, en Newsletter of European Society of Criminology, $\mathrm{n}^{\mathrm{o}}$ 2. Disponible en: http://www.esc-eurocrim.org/newsletter/July12ESCnewsletter.pdf [Consultado por última vez: 17 de septiembre de 2012] 
DÍEZ RIPOLLÉS, José Luis (2003): La racionalidad de las leyes penales. Madrid, Trotta.

GARCÍA ESPAÑA, Elisa (2001): Inmigración y delincuencia en España: Análisis criminológico. Valencia, Tirant lo Blanch.

GARCÍA ESPAÑA, Elisa y DÍEZ RIPOLLES, José Luis. (2009): Encuesta a víctimas en España. Sevilla, IAIC-Cajasol.

GARCÍA ESPAÑA, Elisa y DÍEZ RIPOLLÉS, José Luis (2012): Realidad y política penitenciarias. Valencia, Tirant lo Blanch-IAIC.

GÓMEZ FRAGUELA y otros (2009): "El mito del inmigrante delincuente". Boletín Criminológico, $\mathrm{n}^{\circ}$ 112. Disponible en: http://www.boletincriminologico.uma.es/boletines/112.pdf [Consultado por última vez: 21 de septiembre de 2012]

GUANIPA-PÉREZ, María (2012): "Elementos del proceso de comunicación que orientan el discurso de los profesores universitarios". Revista de Comunicación Vivat Academia, $\mathrm{n}^{\mathbf{0}}$ 118, Marzo 2012. Madrid. Universidad Complutense, recuperado el 8 de noviembre de 2012, en la siguiente dirección: http://www.ucm.es/info /vivataca/numeros/n118/DATOSS.htm

FERNÁNDEZ MOLINA, Esther y otros (2009): "Evolución y tendencias de la delincuencia juvenil en España". REIC $\mathrm{n}^{\circ} 7$. Disponible en: http://www.criminologia.net/pdf/reic/ano7-2009/a72009art8.pdf. [Consultado por última vez: 20 de septiembre de 2012]

MARTEACHE SOLANS, Nerea y otros (2010): “Comparación entre opinión pública y "opinión pública meditada" en relación a la delincuencia sexual". REIC n ${ }^{\circ} 8$. Disponible en: http://www.criminologia.net/pdf/reic/ano8-2010/a82010art7.pdf. [Consultado por última vez: 20 de septiembre de 2012]

MARTÍNEZ RODRÍGUEZ, Beatriz (2012): "Periodismo y responsabilidad social: el paradigma del tratamiento informativo de la violencia contra la mujer". Revista de Comunicación Vivat Academia, $\mathrm{n}^{\mathrm{o}}$ 119, junio 2012. Madrid. Universidad Complutense, recuperado el 8 de noviembre de 2012, en la siguiente dirección: http://www.ucm.es/info/vivataca/numeros/n119/DATOSS119.htm

MUT CAMACHO, Magdalena (2012): "Apuntes sobre la opinión pública a pie de calle". Revista de Comunicación SEECI, n ${ }^{\circ} 28$, Julio 2012. Madrid. Universidad Complutense, recuperado el 8 de noviembre de 2012, en la siguiente dirección: http://www.ucm.es/info/seeci/Numeros/Numero28/DATOS28.html

REDONDO ILLESCAS, Santiago (2006): ¿Sirve el tratamiento para rehabilitar a los delincuentes? REIC, $\mathrm{n}^{\circ}$ 4. Disponible en http://www.criminologia.net/pdf/reic /ano4-2006/a42006art3.pdf.

\section{Elisa GARCÍA ESPÃ̃̃}

Universidad de Málaga

Facultad de Derecho

Profesora Titular de Criminología y Derecho penal

elisa@uma.es 